\title{
Biorthogonal systems in Banach spaces
}

\author{
by \\ Michael A. Coco (Lynchburg, VA)
}

\begin{abstract}
We give biorthogonal system characterizations of Banach spaces that fail the Dunford-Pettis property, contain an isomorphic copy of $c_{0}$, or fail the hereditary Dunford-Pettis property. We combine this with previous results to show that each infinitedimensional Banach space has one of three types of biorthogonal systems.
\end{abstract}

1. Introduction. When we first encounter an arbitrary Banach space, we usually search for some kind of fundamental structure in the space to make our understanding of it more complete. Very often, if a space has (or fails) a certain property, we can find a fundamental structure within the space that reflects the property (or failure thereof). Of course, in this case, we would like to find a strong structure, like a Schauder basis or finitedimensional decomposition (FDD), in the space. However, this is not always possible, as even a separable Banach space need not contain a Schauder basis [8]. For this reason it is interesting to consider weaker structures than FDD's and Schauder bases which exist in every separable Banach space and try to prove that a separable Banach space has a certain property if and only if there is a structure in the space which reflects the property.

One useful basis-like structure that has been considered for a long time is that of fundamental total biorthogonal system. Markushevich [11] showed in 1943 that each separable Banach space contains a fundamental total biorthogonal system. The main theorems of the present paper give a biorthogonal system characterization of spaces failing the Dunford-Pettis property and spaces containing an isomorphic copy of $c_{0}$. Combining this with work already done in the field yields a theorem about the existence of biorthogonal systems in any given infinite-dimensional Banach space.

2000 Mathematics Subject Classification: Primary 46B20, 46B25, 46B15.

This work is part of the author's $\mathrm{PhD}$ dissertation under the direction of Dr. Maria K. Girardi at the University of South Carolina. Special thanks to her for all her guidance and inspiration.

The author also wishes to thank Dr. Joseph Diestel at Kent State University who had a profound effect on this work. 
2. Notation and motivation. Throughout this paper, $\mathfrak{X}$ denotes an arbitrary (infinite-dimensional real) Banach space. If $\mathfrak{X}$ is a Banach space, then $\mathfrak{X}^{*}$ is its topological dual space, $B(\mathfrak{X})$ is its (closed) unit ball, and $S(\mathfrak{X})$ is its unit sphere. If $X$ is a subset of $\mathfrak{X}$, then $\operatorname{sp}\{X\}$ is the linear span of $X$ while $[X]$ is the closed linear span of $X$. The Kronecker delta $\delta_{n m}$ takes the value 1 when $n=m$ and 0 when $n \neq m$.

Definition 2.1. For a subset $X$ of $\mathfrak{X}$ and a subset $Z$ of $\mathfrak{X}^{*}$ :

(1) the annihilator of $X$ is $X^{\perp}=\left\{x^{*} \in \mathfrak{X}^{*}: x^{*}(x)=0\right.$ for all $\left.x \in X\right\}$,

(2) the preannihilator of $Z$ is $Z^{\top}=\left\{x \in \mathfrak{X}: x^{*}(x)=0\right.$ for all $\left.x^{*} \in Z\right\}$,

(3) $X$ is fundamental if $[X]=\mathfrak{X}$, or equivalently, $X^{\perp}=\{0\}$,

(4) $Z$ is total if the weak ${ }^{*}$-closure of $\operatorname{sp}\{Z\}$ is $\mathfrak{X}^{*}$, or equivalently, $Z^{\top}=\{0\}$

(5) for a fixed $\tau \geq 1, Z \tau$-norms $X$ (or $X$ is $\tau$-normed by $Z$ ) if

$$
\|x\| \leq \tau \sup _{z \in Z \backslash\{0\}} \frac{z(x)}{\|z\|}
$$

for each $x \in X$,

(6) $Z$ norms $X$ if $Z$ 1-norms $X$.

It is easy to see that if $Z \tau$-norms $\mathfrak{X}$ for a $\tau \geq 1$ then $Z$ is total.

Definition 2.2. A system $\left\{x_{n}, x_{n}^{*}\right\}_{n=1}^{\infty}$ in $X \times Z$ is

(1) a biorthogonal system if $x_{n}^{*}\left(x_{m}\right)=\delta_{n m}$,

(2) $M$-bounded if $\left\{x_{n}\right\}$ and $\left\{x_{n}^{*}\right\}$ are bounded and $\sup _{n}\left\|x_{n}\right\|\left\|x_{n}^{*}\right\| \leq M$,

(3) bounded if it is $M$-bounded for some (finite) $M$,

(4) fundamental if $\left\{x_{n}\right\}$ is fundamental,

(5) total if $\left\{x_{n}^{*}\right\}$ is total.

A sequence $\left\{x_{n}\right\}_{n=1}^{\infty}$ in a Banach space $\mathfrak{X}$ is called semi-normalized if there are constants $0<\alpha \leq \beta<\infty$ such that $\alpha \leq\left\|x_{n}\right\| \leq \beta$ for each $n \in \mathbb{N}$. Recall that $\left\{x_{n}\right\}_{n=1}^{\infty}$ is a basic sequence if each $x_{n}$ is non-zero and there exists a finite constant $K>0$ such that

$$
\left\|\sum_{j=1}^{m} a_{j} x_{j}\right\| \leq K\left\|\sum_{j=1}^{n} a_{j} x_{j}\right\|
$$

for all choices $\left\{a_{j}\right\}_{j \in \mathbb{N}}$ and any integers $m<n$. When this is the case, the smallest $K$ for which (2.1) holds is called the basis constant of $\left\{x_{n}\right\}_{n=1}^{\infty}$ and there exists a biorthogonal system $\left\{x_{n}, x_{n}^{*}\right\}$ in $\mathfrak{X} \times \mathfrak{X}^{*}$ such that $\left\|x_{n}^{*}\right\| \leq$ $2 K /\left\|x_{n}\right\|$.

Operators between Banach spaces are assumed to be bounded and linear. All notation and terminology, not otherwise explained, are as in [6] or [10].

Our motivation begins with the following structure theorem of E. Odell [12]: 
THEOREM 2.3. Every infinite-dimensional Banach space contains a subspace isomorphic to $c_{0}$, a subspace isomorphic to $\ell_{1}$ or a subspace that fails the Dunford-Pettis property.

Our goal is to find a biorthogonal system version of this theorem in which the conditions imposed on the biorthogonal systems directly reflect the property they characterize. Luckily, some of the work, the $\ell_{1}$ case, has already been done for us. In fact, our results are inspired by this previous work. In 2000, S. J. Dilworth, M. Girardi, and W. B. Johnson characterized spaces containing isomorphic copies of $\ell_{1}$ using biorthogonal systems.

THEOREM 2.4 ([7]). The following statements are equivalent:

(1) $\ell_{1} \hookrightarrow \mathfrak{X}$.

(2) There is a bounded $w c_{0}^{*}$-stable biorthogonal system in $\mathfrak{X} \times \mathfrak{X}^{*}$. And in the case that $\mathfrak{X}$ is separable:

(3) There is a bounded fundamental total $w c_{0}^{*}$-stable biorthogonal system $\left\{x_{n}, x_{n}^{*}\right\}$ in $\mathfrak{X} \times \mathfrak{X}^{*}$.

Furthermore for each $\varepsilon>0$ : if (2) holds then the system can be taken to be $(1+\varepsilon)$-bounded; if $(3)$ holds then the system can be taken to be $[(1+\sqrt{2})+\varepsilon]$ bounded and so that $\left[x_{n}^{*}\right](2+\varepsilon)$-norms $\mathfrak{X}$.

Recall that $\left\{x_{n}, x_{n}^{*}\right\}$ is a $w c_{0}^{*}$-stable biorthogonal system if, for each isomorphic embedding $T$ of $\mathfrak{X}$ into some $\mathcal{Y}$, there exists a lifting $\left\{y_{n}^{*}\right\}$ of $\left\{x_{n}^{*}\right\}$ (i.e., $T^{*} y_{n}^{*}=x_{n}^{*}$ for each $n$ ) such that $\left\{y_{n}^{*}\right\}$ is a semi-normalized weakly null sequence in $\mathcal{Y}^{*}$ (or equivalently, such that $\left\{T x_{n}, y_{n}^{*}\right\}$ in $\mathcal{Y} \times \mathcal{Y}^{*}$ is a $w c_{0}^{*}$-biorthogonal system).

They also characterized Banach spaces that have the Schur property (i.e. weak and strong sequential convergence in $\mathfrak{X}$ coincide) via biorthogonal systems. In the next section we will discuss the Dunford-Pettis property. Recall that the Schur property is related to the Dunford-Pettis property and embeddings of $\ell_{1}$ in the following way (cf. [4, p. 23]): $\mathfrak{X}^{*}$ fails the Schur property if and only if $\mathfrak{X}$ fails the Dunford-Pettis property or $\ell_{1} \hookrightarrow \mathfrak{X}$. This fact provides a link between the above results and the results of the next section that characterize failure of the Dunford-Pettis property.

3. Spaces failing the Dunford-Pettis property. Recall that a Banach space $\mathfrak{X}$ has the Dunford-Pettis property (DP) if whenever $\left\{x_{n}\right\}_{n} \subset \mathfrak{X}$ and $\left\{x_{n}^{*}\right\}_{n} \subset \mathfrak{X}^{*}$ are weakly null sequences, we have $\lim _{n \rightarrow \infty} x_{n}^{*}\left(x_{n}\right)=0$. We refer the reader to the excellent survey article [4] for a complete treatment of all things Dunford-Pettis. Further results and additional open questions can be found in [2].

Now suppose $\mathfrak{X}$ is a Banach space that fails the Dunford-Pettis property. Then there exists a weakly null sequence $\left\{w_{k}\right\}_{k \in \mathbb{N}}$ in $\mathfrak{X}$ and a weakly null 
sequence $\left\{w_{k}^{*}\right\}_{k \in \mathbb{N}}$ in $\mathfrak{X}^{*}$ such that $\lim _{k \rightarrow \infty}\left|w_{k}^{*}\left(w_{k}\right)\right| \neq 0$. We may assume, without loss of generality, that there exists $\delta>0$ such that $w_{k}^{*}\left(w_{k}\right)>\delta$ for each $k \in \mathbb{N}$. If this is not the case we can pass to a suitable subsequence and adjust signs. Now $\left\{w_{k}\right\}_{k \in \mathbb{N}}$ and $\left\{w_{k}^{*}\right\}_{k \in \mathbb{N}}$ are semi-normalized so we may renormalize if necessary to get for each $k \in \mathbb{N}$ :

(1) $w_{k} \in S(\mathfrak{X})$,

(2) $w_{k}^{*}\left(w_{k}\right)=1$,

(3) $1 \leq\left\|w_{k}^{*}\right\| \leq M$ for some constant $M$.

This leads to the following definition.

Definition 3.1. Let $M \geq 1$. Then $\mathfrak{X}$ fails the $M$-Dunford-Pettis property $(M-\mathrm{DP})$ provided there is a weakly null sequence $\left\{w_{k}\right\}_{k}$ from $S(\mathfrak{X})$ and a weakly null sequence $\left\{w_{k}^{*}\right\}_{k}$ from $\mathfrak{X}^{*}$ such that $w_{k}^{*}\left(w_{k}\right)=1$ and $1 \leq\left\|w_{k}^{*}\right\| \leq M$ for each $k \in \mathbb{N}$.

Note that clearly $\mathfrak{X}$ fails $M$-DP for some $M$ if and only if $\mathfrak{X}$ fails DP. We only bother to define it here to make the statement of Theorem 3.3 a bit clearer.

Definition 3.2. A biorthogonal system $\left\{x_{n}, x_{n}^{*}\right\}$ in $\mathfrak{X} \times \mathfrak{X}^{*}$ is called a DP-biorthogonal system if $\left\{x_{n}\right\}$ and $\left\{x_{n}^{*}\right\}$ are semi-normalized weakly-null sequences.

THEOREM 3.3. The following statements are equivalent:

(1) $\mathfrak{X}$ fails the Dunford-Pettis property.

(2) There is a bounded DP-biorthogonal system in $\mathfrak{X} \times \mathfrak{X}^{*}$. And in the case that $\mathfrak{X}$ is separable:

(3) There is a bounded fundamental total DP-biorthogonal system $\left\{x_{n}, x_{n}^{*}\right\}$ in $\mathfrak{X} \times \mathfrak{X}^{*}$.

Furthermore, for an $\mathfrak{X}$ failing the $M$-Dunford-Pettis property, for each $\varepsilon>0$ : if (2) holds then the system can be taken to be $(M+\varepsilon)$-bounded; if (3) holds then the system can be taken to be $\left[M(1+\sqrt{2})^{2}+\varepsilon\right]$-bounded and so that $\left[x_{n}^{*}\right]$ norms $\mathfrak{X}$.

It is clear that (2) implies (1) as well as (3) implies (1). That (1) implies (2) follows from Theorem 3.6. That (1) implies (3) in the separable case follows from Theorem 3.9.

The following well known basic facts will be used.

FACT 3.4. If $\left\{x_{n}\right\}_{n}$ is weakly null and $\varliminf_{n}\left\|x_{n}\right\|>0$ and $\varepsilon>0$, then $\left\{x_{n}\right\}_{n}$ has a subsequence which is a basic sequence with basis constant at most $1+\varepsilon$. 
FACT 3.5. Let $\mathfrak{X}_{0}$ be a finite-codimensional subspace of $\mathfrak{X}$ and $\left\{x_{n}\right\}_{n \in \mathbb{N}}$ be a weakly null sequence in $\mathfrak{X}$. Then

$$
d\left(x_{n}, \mathfrak{X}_{0}\right):=\inf _{x_{0} \in \mathfrak{X}_{0}}\left\|x_{n}-x_{0}\right\| \stackrel{n \rightarrow \infty}{\longrightarrow} 0 .
$$

Thus, if $\left\{x_{n}\right\}_{n}$ is semi-normalized and $\varepsilon>0$, there exist $n_{\varepsilon}$ and $\widetilde{x}_{n_{\varepsilon}} \in \mathfrak{X}_{0}$ with $\left\|x_{n_{\varepsilon}}-\widetilde{x}_{n_{\varepsilon}}\right\|<\varepsilon$ and $\left\|x_{n_{\varepsilon}}\right\|=\left\|\widetilde{x}_{n_{\varepsilon}}\right\|$.

We can now give a quantitative proof that (1) implies (2) in Theorem 3.3.

TheOREM 3.6. Let $\mathfrak{X}$ fail the $M$-Dunford-Pettis property and $\varepsilon>0$. Then there is a biorthogonal system $\left\{x_{n}, x_{n}^{*}\right\}_{n=1}^{\infty}$ in $\mathfrak{X} \times \mathfrak{X}^{*}$ such that:

(1) $\left\{x_{n}\right\}_{n=1}^{\infty}$ and $\left\{x_{n}^{*}\right\}_{n=1}^{\infty}$ are weakly null,

(2) $\left\|x_{n}\right\|=1$ for each $n \in \mathbb{N}$,

(3) $1 \leq\left\|x_{n}^{*}\right\| \leq M+\varepsilon$ for each $n \in \mathbb{N}$,

(4) $\left\{x_{n}\right\}_{n=1}^{\infty}$ is a basic sequence.

Proof. Since $\mathfrak{X}$ fails the $M$-Dunford-Pettis property there exist sequences $\left\{w_{k}\right\}_{k \in \mathbb{N}}$ and $\left\{w_{k}^{*}\right\}_{k \in \mathbb{N}}$ as in Definition 3.1. By Fact 3.4 we may assume $\left\{w_{k}\right\}_{k \in \mathbb{N}}$ is a basic sequence.

Let $\left\{\varepsilon_{n}\right\}_{n \in \mathbb{N}}$ be a decreasing sequence of positive numbers with $\varepsilon_{1}<$ $\varepsilon / 2(M+\varepsilon)$ and $\sum_{n \in \mathbb{N}} \varepsilon_{n}<1 / 2 K$ where $K$ is the basis constant of $\left\{w_{k}\right\}_{k \in \mathbb{N}}$. We will construct a system $\left\{x_{n}, x_{n}^{*}\right\}_{n=1}^{\infty}$ in $\mathfrak{X} \times \mathfrak{X}^{*}$ and an increasing sequence $\left\{k_{n}\right\}_{n \geq 1}$ of integers such that

(a) $\left\{x_{n}, x_{n}^{*}\right\}_{n=1}^{\infty}$ is biorthogonal,

(b) $\left\|x_{n}\right\|=1$ for each $n \in \mathbb{N}$,

(c) $1 \leq\left\|x_{n}^{*}\right\| \leq M /\left(1-2 \varepsilon_{n}\right)$ for each $n \in \mathbb{N}$,

(d) $\left\|x_{n}-w_{k_{n}}\right\| \leq \varepsilon_{n} / M$ for each $n \in \mathbb{N}$,

(e) $\left\|x_{n}^{*}-w_{k_{n}}^{*}\right\| \leq \varepsilon_{n}+2 M \varepsilon_{n} /\left(1-2 \varepsilon_{n}\right)$ for each $n \in \mathbb{N}$.

Conditions (d) and (e) will give us (1): for $x^{*} \in \mathfrak{X}^{*}$,

$$
\left|x^{*}\left(x_{n}\right)\right| \leq\left\|x^{*}\right\|\left\|x_{n}-w_{k_{n}}\right\|+\left|x^{*}\left(w_{k_{n}}\right)\right| \rightarrow 0
$$

so $\left\{x_{n}\right\}_{n}$ is weakly null and similarly for $\left\{x_{n}^{*}\right\}_{n}$.

Condition (c) gives us (3):

$$
1 \leq\left\|x_{n}^{*}\right\| \leq \frac{M}{1-2 \varepsilon_{n}} \leq \frac{M}{1-2\left(\frac{\varepsilon}{2(M+\varepsilon)}\right)}=M+\varepsilon .
$$

Condition (d) gives us (4): we have

$$
\sum_{n}\left\|w_{k_{n}}-x_{n}\right\| \leq \sum_{n} \varepsilon_{n}<\frac{1}{2 K} .
$$

Then $\left\{x_{n}\right\}_{n}$ is basic (and equivalent to $\left\{w_{n_{k}}\right\}_{k}$ ).

Now we construct $\left\{x_{n}, x_{n}^{*}\right\}_{n=1}^{\infty}$ by induction. To start, let $k_{1}=1$ and $x_{1}=w_{1}$ and $x_{1}^{*}=w_{1}^{*}$. Fix $n>1$ and assume that a system $\left\{x_{j}, x_{j}^{*}\right\}_{j<n}$ 
along with a sequence $\left\{k_{j}\right\}_{j<n}$ have been constructed to satisfy the above conditions. Let

$$
\mathfrak{X}_{n}=\left[x_{j}^{*}\right]_{j<n}^{\top}, \quad \mathcal{Z}_{n}=\left[x_{j}\right]_{j<n}^{\perp} .
$$

Using Fact 3.5, find $k_{n}>k_{n-1}$ and $x_{n} \in \mathfrak{X}_{n}$ and $z_{n}^{*} \in \mathcal{Z}_{n}$ so that

$$
d\left(w_{k_{n}}, \mathfrak{X}_{n}\right) \leq\left\|w_{k_{n}}-x_{n}\right\|<\varepsilon_{n} / M, \quad d\left(w_{k_{n}}^{*}, \mathcal{Z}_{n}\right) \leq\left\|w_{k_{n}}^{*}-z_{n}^{*}\right\|<\varepsilon_{n}
$$

with $\left\|x_{n}\right\|=1$ and $1 \leq\left\|z_{n}^{*}\right\| \leq M$. Note that

$$
\begin{aligned}
\left|z_{n}^{*}\left(x_{n}\right)-w_{k_{n}}^{*}\left(w_{k_{n}}\right)\right| & =\left|z_{n}^{*}\left(x_{n}-w_{k_{n}}\right)-\left(w_{k_{n}}^{*}-z_{n}^{*}\right)\left(w_{k_{n}}\right)\right| \\
& <M \frac{\varepsilon_{n}}{M}+\varepsilon_{n}=2 \varepsilon_{n},
\end{aligned}
$$

and so $1-2 \varepsilon_{n}<z_{n}^{*}\left(x_{n}\right)<1+2 \varepsilon_{n}$. Let

$$
x_{n}^{*}:=\frac{z_{n}^{*}}{z_{n}^{*}\left(x_{n}\right)} .
$$

Thus conditions (a) and (c) hold. As for condition (e):

$$
\begin{aligned}
\left\|x_{n}^{*}-w_{k_{n}}^{*}\right\| & \leq\left\|w_{k_{n}}^{*}-z_{n}^{*}\right\|+\left\|z_{n}^{*}-\frac{z_{n}^{*}}{z_{n}^{*}\left(x_{n}\right)}\right\| \\
& \leq \varepsilon_{n}+\frac{1}{z_{n}^{*}\left(x_{n}\right)}\left|z_{n}^{*}\left(x_{n}\right)-1\right|\left\|z_{n}^{*}\right\| \leq \varepsilon_{n}+\frac{2 \varepsilon_{n}}{1-2 \varepsilon} M .
\end{aligned}
$$

The constructions of fundamental total biorthogonal systems in the proofs of $(1) \Rightarrow(3)$ in Theorems 3.3 and 4.5 use the Haar matrices, which are summarized below.

Remark 3.7. Fix $m \geq 0$ and consider the $2^{m}$-dimensional Hilbert space $\ell_{2}^{2^{m}}$, along with its unit vector basis $\left\{e_{j}^{2}\right\}_{j=1}^{2^{m}}$.

The Haar basis $\left\{h_{j}^{m}\right\}_{j=1}^{2^{m}}$ of $\ell_{2}^{2^{m}}$ can be described as follows. For $0 \leq n$ $\leq m$ and $1 \leq k \leq 2^{n}$ let

$$
I_{k}^{n}=\left\{j \in \mathbb{N}: 2^{m-n}(k-1)<j \leq 2^{m-n} k\right\} .
$$

Thus

$$
I_{1}^{0}=\left\{1,2, \ldots, 2^{m}\right\}, \quad I_{1}^{1}=\left\{1,2, \ldots, 2^{m-1}\right\}, \quad I_{1}^{1}=\left\{1+2^{m-1}, \ldots, 2^{m}\right\} .
$$

In general, the collection $\left\{I_{k}^{n}\right\}_{k=1}^{2^{n}}$ of sets along the $n$th level (disjointly) partitions the set $\left\{1,2, \ldots, 2^{m}\right\}$ into $2^{n}$ sets, each containing $2^{m-n}$ consecutive integers, and $I_{k}^{n}$ is the disjoint union $I_{k}^{n}=I_{2 k-1}^{n+1} \cup I_{2 k}^{n+1}$. Now let

$$
h_{1}^{m}=2^{-m / 2} \sum_{j \in I_{1}^{0}} e_{j}^{2}
$$

and, for $0 \leq n<m$ and $1 \leq k \leq 2^{n}$, let $h_{2^{n}+k}^{m}$ be supported on $I_{k}^{n}$ as

$$
h_{2^{n}+k}^{m}=2^{(n-m) / 2}\left[\sum_{j \in I_{2 k-1}^{n+1}} e_{j}^{2}-\sum_{j \in I_{2 k}^{n+1}} e_{j}^{2}\right] .
$$

Note that $\left\{h_{j}^{m}\right\}_{j=1}^{2^{m}}$ forms an orthonormal basis for $\ell_{2}^{2^{m}}$. 
Let $H_{m}=\left(a_{i j}^{m}\right)$ be the $2^{m} \times 2^{m}$ Haar matrix that transforms the unit vector basis of $\ell_{2}^{2^{m}}$ onto the Haar basis; thus, the $j$ th column vector of $H_{m}$ is just $h_{j}^{m}$ and so $H_{m}$ is a unitary matrix. For example, for $m=2$ we have

$$
H_{2}=\left[\begin{array}{cccc}
2^{-1} & +2^{-1} & +2^{-1 / 2} & 0 \\
2^{-1} & +2^{-1} & -2^{-1 / 2} & 0 \\
2^{-1} & -2^{-1} & 0 & +2^{-1 / 2} \\
2^{-1} & -2^{-1} & 0 & -2^{-1 / 2}
\end{array}\right]
$$

Now if $\left\{z_{j}, z_{j}^{*}\right\}_{j=1}^{2^{m}}$ is a biorthogonal sequence in $\mathfrak{X} \times \mathfrak{X}^{*}$ and $\left\{x_{i}, x_{i}^{*}\right\}_{i=1}^{2^{m}}$ is such that

$$
H_{m}\left[\begin{array}{c}
z_{1} \\
\vdots \\
z_{2^{m}}
\end{array}\right]=\left[\begin{array}{c}
x_{1} \\
\vdots \\
x_{2^{m}}
\end{array}\right], \quad H_{m}\left[\begin{array}{c}
z_{1}^{*} \\
\vdots \\
z_{2^{m}}^{*}
\end{array}\right]=\left[\begin{array}{c}
x_{1}^{*} \\
\vdots \\
x_{2^{m}}^{*}
\end{array}\right]
$$

then

$$
x_{i}:=\sum_{j=1}^{2^{m}} a_{i j}^{m} z_{j}, \quad x_{i}^{*}:=\sum_{j=1}^{2^{m}} a_{i j}^{m} z_{j}^{*} .
$$

It is not hard to see that since $H_{m}$ is a unitary matrix,

(1) $x_{i}^{*}\left(x_{j}\right)=\delta_{i j}$,

(2) $\left[x_{i}\right]_{i=1}^{2^{m}}=\left[z_{j}\right]_{j=1}^{2^{m}}$,

(3) $\left[x_{i}^{*}\right]_{i=1}^{2^{m}}=\left[z_{j}^{*}\right]_{j=1}^{2^{m}}$.

Note that, for each $1 \leq i \leq 2^{m}$,

(4) $a_{i 1}^{m}=2^{-m / 2}$,

(5) $\sum_{j=2}^{2^{m}}\left|a_{i j}^{m}\right|=(1+\sqrt{2})\left(1-2^{-m / 2}\right) \nearrow 1+\sqrt{2}$ as $m \rightarrow \infty$.

It follows that

(6) $\left\|x_{i}\right\| \leq 2^{-m / 2}\left\|z_{1}\right\|+(1+\sqrt{2}) \max _{1<j \leq 2^{m}}\left\|z_{j}\right\|$,

(7) $\left\|x_{i}^{*}\right\| \leq 2^{-m / 2}\left\|z_{1}^{*}\right\|+(1+\sqrt{2}) \max _{1<j \leq 2^{m}}\left\|z_{j}^{*}\right\|$,

(8) for each $x^{*} \in \mathfrak{X}^{*}$,

$$
\left|x^{*}\left(x_{i}\right)\right| \leq 2^{-m / 2}\left|x^{*}\left(z_{1}\right)\right|+(1+\sqrt{2}) \max _{1<j \leq 2^{m}}\left|x^{*}\left(z_{j}\right)\right|,
$$

(9) for each $x^{* *} \in \mathfrak{X}^{* *}$,

$$
\left|x^{* *}\left(x_{i}^{*}\right)\right| \leq 2^{-m / 2}\left|x^{* *}\left(z_{1}^{*}\right)\right|+(1+\sqrt{2}) \max _{1<j \leq 2^{m}}\left|x^{* *}\left(z_{j}^{*}\right)\right| .
$$

The following notation will (hopefully) simplify the proofs of Theorem 3.9 and Theorem 4.8 . 
Definition 3.8. A sequence $\left\{J_{k}\right\}_{k=1}^{\infty}$ of subsets of $\mathbb{N}$ is a blocking of $\mathbb{N}$ if $\mathbb{N}$ is the disjoint union $\bigcup_{k=1}^{\infty} J_{k}$ and

$$
\max J_{k}<\min J_{k+1}
$$

for each $k \in \mathbb{N}$. Given a blocking $\left\{J_{k}\right\}_{k=1}^{\infty}$ of $\mathbb{N}$, let $J_{0}=\emptyset$ and

$$
\begin{aligned}
J_{k}^{p} & :=\bigcup_{0 \leq j<k} J_{j}, \quad J_{k}^{o}:=J_{k} \backslash\left\{\text { the first element in } J_{k}\right\} \\
J_{k}^{p o} & :=\bigcup_{0 \leq j<k} J_{j}^{o}, \quad \mathbb{N}^{o}:=\bigcup_{k=1}^{\infty} J_{k}^{o}
\end{aligned}
$$

for each $k \in \mathbb{N}$. Pictorially one has:

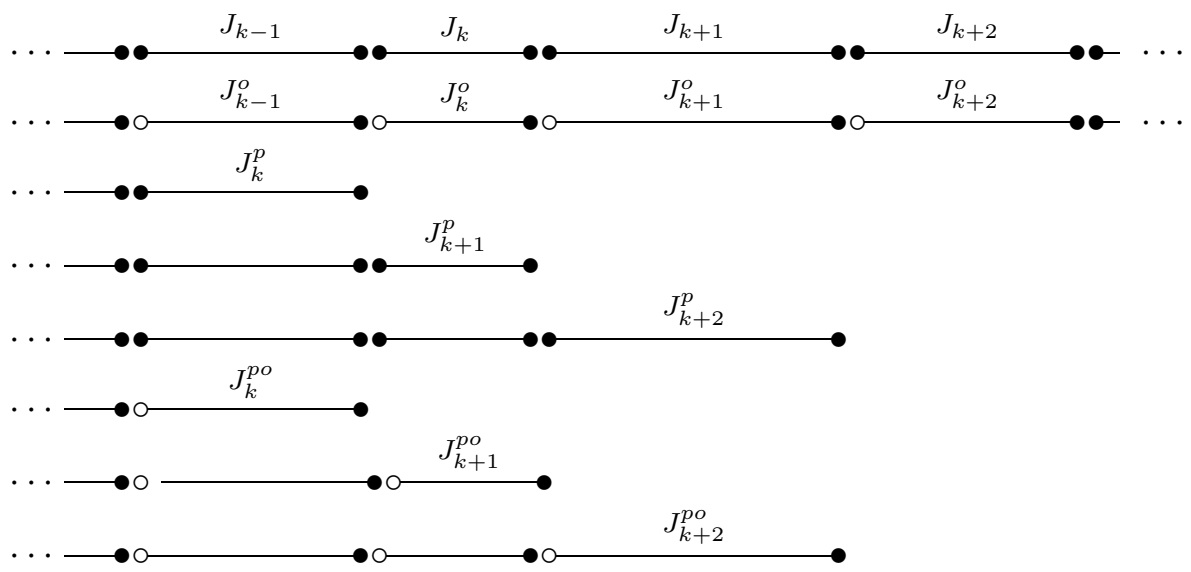

It follows from the next theorem that (1) implies (3) for separable $\mathfrak{X}$ in Theorem 3.3.

TheOrem 3.9. Let $\mathfrak{X}$ fail the $M$-Dunford-Pettis property and $\varepsilon>0$. If $\left\{a_{n}, b_{n}^{*}\right\}_{n \in \mathbb{N}} \subset \mathfrak{X} \times \mathfrak{X}^{*}$ then there exists an $\left[M(1+\sqrt{2})^{2}+\varepsilon\right]$-bounded DP-biorthogonal system $\left\{x_{n}, x_{n}^{*}\right\}$ in $\mathfrak{X} \times \mathfrak{X}^{*}$ such that $\left[a_{n}\right]_{n \in \mathbb{N}} \subset\left[x_{n}\right]_{n \in \mathbb{N}}$ and $\left[b_{n}^{*}\right]_{n \in \mathbb{N}} \subset\left[x_{n}^{*}\right]_{n \in \mathbb{N}}$.

Proof. Without loss of generality, $\left[a_{n}\right]_{n \in \mathbb{N}}$ and $\left[b_{n}^{*}\right]_{n \in \mathbb{N}}$ are each infinitedimensional. Since $\mathfrak{X}$ fails the $M$-Dunford-Pettis property, by Theorem 3.6, there is a biorthogonal system $\left\{w_{n}, w_{n}^{*}\right\}$ in $\mathfrak{X} \times \mathfrak{X}^{*}$ with both $\left\{w_{n}\right\}_{n}$ and $\left\{w_{n}^{*}\right\}_{n}$ weakly null, $\left\|w_{n}\right\|=1$, and $1 \leq\left\|w_{n}^{*}\right\| \leq M+\varepsilon$. Fix a sequence $\left\{\delta_{k}\right\}_{k=1}^{\infty}$ of positive numbers decreasing to zero with $\delta_{1}<1 / 2$ and

$$
\frac{M+\varepsilon}{(1+2 \varepsilon) M}<1-2 \delta_{1} \text {. }
$$

It suffices to find a system $\left\{x_{n}, x_{n}^{*}\right\}_{n=1}^{\infty}$ in $\mathfrak{X} \times \mathfrak{X}^{*}$ along with (following the terminology in Definition 3.8) a blocking $\left\{J_{k}\right\}_{k=1}^{\infty}$ of $\mathbb{N}$ and an increasing sequence $\left\{i_{n}\right\}_{n \in \mathbb{N}^{o}}$ from $\mathbb{N}$, satisfying 
(1) $x_{m}^{*}\left(x_{n}\right)=\delta_{m n}$,

(2) $\left\|x_{n}\right\| \leq(1+\sqrt{2})+\varepsilon$,

(3) $\left\|x_{n}^{*}\right\| \leq(1+2 \varepsilon) M(1+\sqrt{2})+\varepsilon$,

(4) for each $x^{*} \in S\left(\mathfrak{X}^{*}\right)$, if $n \in J_{k}$, then

$$
\left|x^{*}\left(x_{n}\right)\right| \leq \delta_{k}+(1+\sqrt{2}) \max _{j \in J_{k}^{o}}\left(\left|x^{*}\left(w_{i_{j}}\right)\right|+\delta_{k}\right),
$$

(5) for each $x^{* *} \in S\left(X^{* *}\right)$, if $n \in J_{k}$ then

$$
\left|x^{* *}\left(x_{n}^{*}\right)\right| \leq \delta_{k}\left(\frac{4+2 M}{1-2 \delta_{k}}\right)+(1+\sqrt{2}) \max _{j \in J_{k}^{o}}\left|x^{* *}\left(w_{i_{j}}^{*}\right)\right|,
$$

(6) $\left[a_{n}\right]_{n=1}^{\infty} \subset\left[x_{n}\right]_{n=1}^{\infty}$,

(7) $\left[b_{n}^{*}\right]_{n=1}^{\infty} \subset\left[x_{n}^{*}\right]_{n=1}^{\infty}$.

The construction will inductively produce blocks $\left\{x_{n}, x_{n}^{*}\right\}_{n \in J_{k}}$. Let $x_{0}$ and $x_{0}^{*}$ be the zero vectors. Fix $k \geq 1$. Assume that $\left\{J_{j}\right\}_{0 \leq j<k}$ along with $\left\{x_{n}, x_{n}^{*}\right\}_{n \in J_{k}^{p}}$ and $\left\{i_{n}\right\}_{n \in J_{k}^{p o}}$ have been constructed to satisfy conditions (1) through (5). Now to construct $J_{k}$ along with $\left\{x_{n}, x_{n}^{*}\right\}_{n \in J_{k}}$ and $\left\{i_{n}\right\}_{n \in J_{k}^{o}}$.

Let

$$
\mathcal{P}_{k}:=\left[x_{n}^{*}\right]_{n \in J_{k}^{p}}^{\top}, \quad \mathcal{Q}_{k}:=\left[x_{n}\right]_{n \in J_{k}^{p}}^{\perp}, \quad n_{k}=\max J_{k}^{p} .
$$

The idea is to find a biorthogonal system $\left\{z_{n}, z_{n}^{*}\right\}_{n \in J_{k}}$ in $\mathcal{P}_{k} \times \mathcal{Q}_{k}$ by first finding just one pair $\left\{z_{1+n_{k}}, z_{1+n_{k}}^{*}\right\}$ which helps guarantee condition (6) if $k$ is odd and condition (7) if $k$ even; however, $\left\{z_{1+n_{k}}, z_{1+n_{k}}^{*}\right\}$ would not necessarily satisfy conditions (2) through (5), and so $J_{k}^{o}$ and $\left\{z_{n}, z_{n}^{*}\right\}_{n \in J_{k}^{o}}$ and $\left\{i_{n}\right\}_{n \in J_{k}^{o}}$ are constructed and then the appropriate Haar matrix is applied to $\left\{z_{n}, z_{n}^{*}\right\}_{n \in J_{k}}$ to produce $\left\{x_{n}, x_{n}^{*}\right\}_{n \in J_{k}}$ so that $\left\{x_{n}, x_{n}^{*}\right\}_{n \in J_{k}^{p} \cup J_{k}}$ with $\left\{i_{n}\right\}_{n \in J_{k}^{p o} \cup J_{k}^{o}}$ satisfy conditions (1) through (5).

The pair $\left\{z_{1+n_{k}}, z_{1+n_{k}}^{*}\right\}$ is constructed by a standard Gram-Schmidt biorthogonal procedure. If $k$ is odd, start in $\mathfrak{X}$. Let

$$
h_{k}=\min \left\{h: a_{h} \notin\left[x_{n}\right]_{n \leq n_{k}}\right\} .
$$

Set

$$
z_{1+n_{k}}=a_{h_{k}}-\sum_{n \leq n_{k}} x_{n}^{*}\left(a_{h_{k}}\right) x_{n}
$$

and for any $y_{1+n_{k}}^{*}$ in $\mathfrak{X}^{*}$ such that $y_{1+n_{k}}^{*}\left(z_{1+n_{k}}\right) \neq 0$,

$$
z_{1+n_{k}}^{*}=\frac{y_{1+n_{k}}^{*}-\sum_{n \leq n_{k}} y_{1+n_{k}}^{*}\left(x_{n}\right) x_{n}^{*}}{y_{1+n_{k}}^{*}\left(z_{1+n_{k}}\right)} .
$$

If $k$ is even, start in $\mathfrak{X}^{*}$. Let

$$
h_{k}=\min \left\{h: b_{h}^{*} \notin\left[x_{n}^{*}\right]_{n \leq n_{k}}\right\} .
$$


Set

$$
z_{1+n_{k}}^{*}=b_{h_{k}}^{*}-\sum_{n \leq n_{k}} b_{h_{k}}^{*}\left(x_{n}\right) x_{n}^{*}
$$

and, for any $y_{1+n_{k}}$ in $\mathfrak{X}$ such that $z_{1+n_{k}}^{*}\left(y_{1+n_{k}}\right) \neq 0$,

$$
z_{1+n_{k}}=\frac{y_{1+n_{k}}-\sum_{n \leq n_{k}} x_{n}^{*}\left(y_{1+n_{k}}\right) x_{n}}{z_{1+n_{k}}^{*}\left(y_{1+n_{k}}\right)} .
$$

Clearly $z_{1+n_{k}}^{*}\left(z_{1+n_{k}}\right)=1, z_{1+n_{k}} \in \mathcal{P}_{k}$ and $z_{1+n_{k}}^{*} \in \mathcal{Q}_{k}$.

Find a natural number $m_{k}$ larger than one so that

$$
2^{-m_{k} / 2} \max \left(\left\|z_{1+n_{k}}\right\|,\left\|z_{1+n_{k}}^{*}\right\|\right)<\min \left(\varepsilon, \delta_{k}\right)
$$

and let

$$
J_{k}:=\left\{1+n_{k}, \ldots, 2^{m_{k}}+n_{k}\right\} \quad \text { and so } \quad J_{k}^{o}:=\left\{2+n_{k}, \ldots, 2^{m_{k}}+n_{k}\right\} .
$$

Let

$$
\widetilde{\mathcal{P}}_{k}:=\mathcal{P}_{k} \cap\left[z_{1+n_{k}}^{*}\right]^{\top}, \quad \widetilde{\mathcal{Q}}_{k}:=\mathcal{Q}_{k} \cap\left[z_{1+n_{k}}\right]^{\perp} .
$$

The next step is to find a biorthogonal system $\left\{z_{n}, z_{n}^{*}\right\}_{n \in J_{k}^{o}}$ along with $\left\{i_{n}\right\}_{n \in J_{k}^{o}}$ satisfying

$$
\left\{z_{n}, z_{n}^{*}\right\} \in S\left(\widetilde{\mathcal{P}}_{k}\right) \times((1+\varepsilon) M) B\left(\widetilde{\mathcal{Q}}_{k}\right)
$$

and

$$
\left\|w_{i_{n}}-z_{n}\right\|<\delta_{k}, \quad\left\|w_{i_{n}}^{*}-z_{n}^{*}\right\|<\delta_{k}+\frac{2 \delta_{k}(M+\varepsilon)}{1-2 \delta_{k}}
$$

for each $n \in J_{k}^{o}$. Towards this, fix $j \in J_{k}^{o}$ and assume that a biorthogonal system $\left\{z_{n}, z_{n}^{*}\right\}_{2+n_{k} \leq n<j}$ along with $\left\{i_{n}\right\}_{2+n_{k} \leq n<j}$ have been constructed so that conditions (3.3) and (3.4) hold for $2+n_{k} \leq n<j$. Let

$$
\mathfrak{X}_{j}:=\widetilde{\mathcal{P}}_{k} \cap\left[z_{n}^{*}\right]_{2+n_{k} \leq n<j}^{\top}, \quad \mathcal{Y}_{j}:=\widetilde{\mathcal{Q}}_{k} \cap\left[z_{n}\right]_{2+n_{k} \leq n<j}^{\perp} .
$$

Then by Fact 3.5 there exists a natural number $i_{j}>i_{j-1}$ along with $z_{j} \in \mathfrak{X}_{j}$ and $\widetilde{z}_{j}^{*} \in \mathcal{Y}_{j}$ such that

$$
\begin{array}{cl}
d\left(w_{i_{j}}, \mathfrak{X}_{j}\right) \leq\left\|w_{i_{j}}-z_{j}\right\|<\frac{\delta_{k}}{M+\varepsilon}, & d\left(w_{i_{j}}^{*}, \mathcal{Y}_{j}\right) \leq\left\|w_{i_{j}}^{*}-\widetilde{z}_{j}^{*}\right\|<\delta_{k}, \\
\left\|z_{j}\right\|=1, & 1 \leq\left\|\widetilde{z}_{j}\right\| \leq M+\varepsilon .
\end{array}
$$

Note that $\widetilde{z}_{j}^{*}\left(z_{j}\right)$ need not be equal to 1 but it is close to 1 since

$$
\begin{aligned}
\left|\widetilde{z}_{j}^{*}\left(z_{j}\right)-w_{i_{j}}^{*}\left(w_{i_{j}}\right)\right| & =\left|\widetilde{z}_{j}^{*}\left(z_{j}\right)-\left(w_{i_{j}}^{*}-\widetilde{z}_{j}^{*}\right)\left(w_{i_{j}}\right)-\widetilde{z}_{j}^{*}\left(w_{i_{j}}\right)\right| \\
& =\left|\widetilde{z}_{j}^{*}\left(z_{j}-w_{i_{j}}\right)-\left(w_{i_{j}}^{*}-\widetilde{z}_{j}^{*}\right)\left(w_{i_{j}}\right)\right| \\
& \leq\left\|\widetilde{z}_{j}^{*}\right\|\left\|z_{j}-w_{i_{j}}\right\|+\left\|w_{i_{j}}^{*}-\widetilde{z}_{j}^{*}\right\|\left\|w_{i_{j}}\right\| \\
& <(M+\varepsilon) \frac{\delta_{k}}{M+\varepsilon}+\delta_{k}=2 \delta_{k}
\end{aligned}
$$


and so $1-2 \delta_{k} \leq \widetilde{z}_{j}^{*}\left(z_{j}\right) \leq 1+2 \delta_{k}$. Let

$$
z_{j}^{*}=\frac{\widetilde{z}_{j}^{*}}{\widetilde{z}_{j}^{*}\left(z_{j}\right)}
$$

so that $z_{j}^{*}\left(z_{j}\right)=1$. Now $z_{j} \in S\left(\widetilde{\mathcal{P}}_{k}\right)$ and $1 \leq\left\|z_{j}^{*}\right\| \leq(M+\varepsilon) /\left(1-2 \delta_{k}\right)$ and so $z_{j}^{*} \in(1+2 \varepsilon) M B\left(\tilde{\mathcal{Q}}_{k}\right)$ by (3.2). Note that by (3.5),

$$
\begin{aligned}
\left\|w_{i_{j}}^{*}-z_{j}^{*}\right\| & \leq\left\|w_{i_{j}}^{*}-\widetilde{z}_{j}^{*}\right\|+\left\|\widetilde{z}_{j}^{*}-z_{j}^{*}\right\| \\
& \leq \delta_{k}+\left\|\widetilde{z}_{j}^{*}\right\|\left|1-\frac{1}{\widetilde{z}_{j}^{*}\left(z_{j}\right)}\right| \\
& \leq \delta_{k}+(1+\varepsilon) M \frac{2 \delta_{k}}{1-2 \delta_{k}} .
\end{aligned}
$$

This completes the inductive construction of $\left\{z_{n}, z_{n}^{*}\right\}_{n \in J_{k}^{o}}$ and $\left\{i_{n}\right\}_{n \in J_{k}^{o}}$.

Now apply the Haar matrix to $\left\{z_{n}, z_{n}^{*}\right\}_{n \in J_{k}}$ to produce $\left\{x_{n}, x_{n}^{*}\right\}_{n \in J_{k}}$. With help from the observations in Remark 3.7, note that $\left\{x_{n}, x_{n}^{*}\right\}_{n \in J_{k}}$ is biorthogonal and is in $\mathcal{P}_{k} \times \mathcal{Q}_{k}$. Furthermore, for each $n$ in $J_{k}$,

$$
\begin{aligned}
& \left\|x_{n}\right\| \leq 2^{-m_{k} / 2}\left\|z_{1+n_{k}}\right\|+(1+\sqrt{2}) \max _{j \in J_{k}^{o}}\left\|z_{j}\right\| \leq \varepsilon+(1+\sqrt{2}), \\
& \left\|x_{n}^{*}\right\| \leq 2^{-m_{k} / 2}\left\|z_{1+n_{k}}^{*}\right\|+(1+\sqrt{2}) \max _{j \in J_{k}^{o}}\left\|z_{j}^{*}\right\| \leq \varepsilon+(1+\varepsilon) M(1+\sqrt{2}) .
\end{aligned}
$$

If $x^{*} \in S\left(\mathfrak{X}^{*}\right)$ then

$$
\begin{aligned}
\left|x^{*}\left(x_{n}\right)\right| & \leq 2^{-m_{k} / 2}\left\|z_{1+n_{k}}\right\|+(1+\sqrt{2}) \max _{j \in J_{k}^{o}}\left|x^{*}\left(z_{j}\right)\right| \\
& \leq \delta_{k}+(1+\sqrt{2}) \max _{j \in J_{k}^{o}}\left(\left|x^{*}\left(w_{i_{j}}\right)\right|+\delta_{k}\right)
\end{aligned}
$$

and for each $x^{* *} \in S\left(\mathfrak{X}^{* *}\right)$,

$$
\begin{aligned}
\left|x^{* *}\left(x_{n}^{*}\right)\right| & \leq 2^{-m_{k} / 2}\left\|z_{1+n_{k}}^{*}\right\|+(1+\sqrt{2}) \max _{j \in J_{k}^{o}}\left|x^{* *}\left(z_{j}^{*}\right)\right| \\
& \leq \delta_{k}+(1+\sqrt{2}) \max _{j \in J_{k}^{o}}\left(\left|x^{* *}\left(w_{i_{j}}^{*}\right)\right|+\delta_{k}+\frac{2 \delta_{k}(1+2 \varepsilon) M}{1-2 \delta_{k}}\right),
\end{aligned}
$$

and this simplifies to give us (5). Thus $\left\{x_{n}, x_{n}^{*}\right\}_{n \in J_{k}^{p} \cup J_{k}}$ with $\left\{i_{n}\right\}_{n \in J_{k}^{p o} \cup J_{k}^{o}}$ satisfy conditions (1) through (5). If $k$ is odd, then

$$
\left[a_{h}\right]_{h \leq h_{k}} \subset\left[x_{n}, z_{1+n_{k}}\right]_{n \in J_{k}^{p}} \subset\left[x_{n}\right]_{n \in J_{k}^{p} \cup J_{k}},
$$

while if $k$ is even, then

$$
\left[b_{h}^{*}\right]_{h \leq h_{k}} \subset\left[x_{n}^{*}, z_{1+n_{k}}^{*}\right]_{n \in J_{k}^{p}} \subset\left[x_{n}^{*}\right]_{n \in J_{k}^{p} \cup J_{k}} .
$$


Clearly the constructed system $\left\{x_{n}, x_{n}^{*}\right\}_{n=1}^{\infty}$ with the blocking $\left\{J_{k}\right\}_{k=1}^{\infty}$ of $\mathbb{N}$ and the increasing sequence $\left\{i_{n}\right\}_{n \in \mathbb{N}^{o}}$ from $\mathbb{N}$ satisfy conditions (1) through (7).

4. Spaces containing $c_{0}$. To motivate the biorthogonal system characterization of spaces containing $c_{0}$ we recall some well known facts about such spaces. We will see that $c_{0}$ subspaces of $\mathfrak{X}$ correspond essentially to weakly unconditionally Cauchy series in $\mathfrak{X}$ so we briefly recall some essential facts about such series.

Definition 4.1. A series $\sum_{n} x_{n}$ is called weakly unconditionally Cauchy (wuC) if given any permutation $\pi$ of $\mathbb{N}$, the sequence $\left\{\sum_{k=1}^{n} x_{\pi(k)}\right\}_{n}$ is weakly Cauchy. Equivalently, $\sum_{n} x_{n}$ is wuC if and only if for each $x^{*} \in \mathfrak{X}^{*}$ we have $\sum_{n}\left|x^{*}\left(x_{n}\right)\right|<\infty$.

Bessaga and Pełczyński [1] tied together wuC series and $c_{0}$.

Theorem 4.2 ([1]). Let $\mathfrak{X}$ be a Banach space.

(1) A basic sequence $\left\{x_{n}\right\}_{n}$ in $\mathfrak{X}$ with $\sum_{n} x_{n}$ wuC and $\inf _{n}\left\|x_{n}\right\|>0$ is equivalent to the unit vector basis of $c_{0}$.

(2) In order that each wuC series $\sum_{n} x_{n}$ in $\mathfrak{X}$ be unconditionally convergent it is both necessary and sufficient that $\mathfrak{X}$ contains no copy of $c_{0}$.

The following ideas will help us define our $c_{0}$-biorthogonal system in a very natural way.

REMARK 4.3. (i) Let $\left\{x_{n}, x_{n}^{*}\right\}$ be a biorthogonal system with $\sum_{n} x_{n}$ wuC and $\underline{\lim }_{n}\left\|x_{n}\right\|>0$. If $\left\{x_{n_{k}}\right\}_{k}$ is any subsequence of $\left\{x_{n}\right\}_{n}$, then $\sum_{k} x_{n_{k}}$ is wuC and $\underline{\lim }_{k}\left\|x_{n_{k}}\right\|>0$ so Fact 3.4 tells us $\left\{x_{n_{k}}\right\}_{k}$ has a subsequence $\left\{x_{n_{k_{j}}}\right\}_{j}$ which is basic and $\inf _{j}\left\|x_{n_{k_{j}}}\right\|>0$. Then by Theorem 4.2, $\left\{x_{n_{k_{j}}}\right\}_{j}$ is equivalent to the unit vector basis of $c_{0}$. Thus each subsequence of $\left\{x_{n}\right\}_{n}$ has a further subsequence which is equivalent to the unit vector basis of $c_{0}$.

(ii) (cf. [5]) Let $T$ be a bounded linear operator from $c_{0}$ to $\mathfrak{X}$ and $x_{n}=$ $T e_{n}$ where $\left\{e_{n}\right\}_{n}$ is the unit vector basis of $c_{0}$. Then for $x^{*} \in \mathfrak{X}^{*}$,

$$
\sum_{n}\left|x^{*}\left(x_{n}\right)\right|=\sum_{n}\left|x^{*}\left(T e_{n}\right)\right|=\sum_{n}\left|T^{*} x^{*}\left(e_{n}\right)\right|<\infty
$$

since $T^{*} x^{*} \in \ell_{1}$. Thus $\sum_{n} x_{n}$ is wuC. Conversely if $\sum_{n} x_{n}$ is wuC in $\mathfrak{X}$, then define $T: c_{0} \rightarrow \mathfrak{X}$ by $T\left(\left\{t_{n}\right\}_{n}\right)=\sum_{n} t_{n} x_{n}$. Then $T$ is well defined and has a closed graph so $T$ is bounded. So the bounded linear operators from $c_{0}$ to $\mathfrak{X}$ correspond precisely to the wuC series in $\mathfrak{X}$.

(iii) Let $T: c_{0} \hookrightarrow \mathfrak{X}$ be an isomorphic embedding and $\left\{e_{n}\right\}_{n}$ be the unit vector basis of $c_{0}$. Since $T$ is an embedding there exist constants $C_{1}$ and $C_{2}$ 
such that for any $\left(\alpha_{n}\right)_{n} \in c_{0}$ we have

$$
C_{1}\left\|\left(\alpha_{n}\right)_{n}\right\|_{c_{0}} \leq\left\|T\left(\left(\alpha_{n}\right)_{n}\right)\right\|_{\mathfrak{X}} \leq C_{2}\left\|\left(\alpha_{n}\right)_{n}\right\|_{c_{0}} .
$$

Then for each $n \in \mathbb{N}$,

$$
C_{1}=C_{1}\left\|e_{n}\right\|_{c_{0}} \leq\left\|T e_{n}\right\|_{\mathfrak{X}} \leq C_{2}\left\|e_{n}\right\|_{c_{0}}=C_{2}
$$

and so $\left\{T e_{n}\right\}_{n}$ is semi-normalized. By (ii) above, the series $\sum_{n} T e_{n}$ is wuC.

Based on this we make the following definition.

Definition 4.4. A biorthogonal system $\left\{x_{n}, x_{n}^{*}\right\}$ in $\mathfrak{X} \times \mathfrak{X}^{*}$ is called a $c_{0}$-biorthogonal system if $\left\{x_{n}\right\}_{n}$ is normalized and has a subsequence $\left\{x_{n_{j}}\right\}_{j}$ for which $\sum_{j} x_{n_{j}}$ is wuC.

THEOREM 4.5. The following statements are equivalent:

(1) $\mathfrak{X}$ contains an isomorphic copy of $c_{0}$.

(2) There is a bounded $c_{0}$-biorthogonal system in $\mathfrak{X} \times \mathfrak{X}^{*}$.

And in the case that $\mathfrak{X}$ is separable:

(3) There is a bounded fundamental total $c_{0}$-biorthogonal system $\left\{x_{n}, x_{n}^{*}\right\}$ $\subset \mathfrak{X} \times \mathfrak{X}^{*}$.

Furthermore, for each $\varepsilon>0$ : if (2) holds then the system can be taken to be $(2+\varepsilon)$-bounded; if clause $(3)$ holds then the system can be taken to be $\left[2(1+\sqrt{2})^{2}+\varepsilon\right]$-bounded and so that $\left[x_{n}^{*}\right]$ norms $\mathfrak{X}$.

That (2) implies (1) as well as (3) implies (1) follow from Remark 4.3. That (1) implies (2) is Theorem 4.6. That (1) implies (3) in the separable case follows from Theorem 4.8 .

THEOREM 4.6. If $\mathfrak{X}$ contains an isomorphic copy of $c_{0}$ and $\varepsilon>0$, then there exists $a(2+\varepsilon)$-bounded $c_{0}$-biorthogonal system $\left\{x_{n}, x_{n}^{*}\right\} \subset S(\mathfrak{X}) \times \mathfrak{X}^{*}$.

Proof. Let $T: c_{0} \hookrightarrow \mathfrak{X}$ be an isomorphic embedding and $\varepsilon>0$. Let $\left\{e_{j}\right\}_{j}$ be the unit vector basis of $c_{0}$. Then Remark 4.3 implies that $\sum_{j} T e_{j}$ is wuC and $\left\{T e_{j}\right\}_{j}$ is semi-normalized. Fact 3.4 gives us a subsequence $\left\{T e_{j_{n}}\right\}_{n}$ of $\left\{T e_{j}\right\}_{j}$ that is basic with basis constant at most $1+\varepsilon / 2$. Let

$$
x_{n}=\frac{T e_{j_{n}}}{\left\|T e_{j_{n}}\right\|} .
$$

Note that $\left\{x_{n}\right\}_{n}$ is a normalized basic sequence with basis constant at most $1+\varepsilon / 2$ and $\sum_{n} x_{n}$ is wuC. We may pick our biorthogonal functionals accordingly.

Notice that the proof of Theorem 4.6 gives us a bit more than a $c_{0^{-}}$ biorthogonal system: it gives us a biorthogonal system $\left\{x_{n}, x_{n}^{*}\right\}$ with the entire series $\sum_{n} x_{n}$ wuC. 
To construct a fundamental total biorthogonal system in the separable case we need the following lemma.

LEMMA 4.7. If $Y_{0}$ is a finite-codimensional subspace of $\mathfrak{X}^{*}$ and $\varepsilon>0$, then there is a finite-codimensional subspace $X_{0}$ of $\mathfrak{X}$ that $i s(2+\varepsilon)$-normed by $Y_{0}$.

Proof. Let $X_{0}$ be the pre-annihilator of any finite-dimensional subspace of $\mathfrak{X}^{*}$ that $(1+\varepsilon)$-norms the annihilator of $Y_{0}$. Then for $f \in S\left(X_{0}\right)$ we have

$$
\begin{aligned}
\sup _{y^{*} \in S\left(Y_{0}\right)}\left|y^{*}(f)\right| & =\inf _{y^{* *} \in Y_{0}^{\perp}}\left\|f-y^{* *}\right\| \\
& \geq \inf _{y^{* *} \in Y_{0}^{\perp}} \max \left[\|f\|-\left\|y^{* *}\right\|, \sup _{x^{*} \in S\left(X_{0}^{\perp}\right)}\left|\left(f-y^{* *}\right)\left(x^{*}\right)\right|\right] \\
& \geq \inf _{y^{* *} \in Y_{0}^{\perp}} \max \left[1-\left\|y^{* *}\right\|, \frac{1}{1+\varepsilon}\left\|y^{* *}\right\|\right] \\
& =\inf _{0 \leq t<\infty} \max \left[1-t, \frac{t}{1+\varepsilon}\right]=\frac{1}{2+\varepsilon} .
\end{aligned}
$$

So $\|f\| \leq(2+\varepsilon) \sup _{y^{*} \in S\left(Y_{0}\right)}\left|y^{*}(f)\right|$ for each $f \in S\left(X_{0}\right)$. Thus $X_{0}$ is $(2+\varepsilon)$-normed by $Y_{0}$.

The following theorem will give us a fundamental total $c_{0}$-biorthogonal system in the separable case.

TheOREM 4.8. Suppose $\mathfrak{X}$ has a subspace isomorphic to $c_{0}$. Let $\varepsilon>0$ and $\left\{a_{n}, b_{n}^{*}\right\} \subset \mathfrak{X} \times \mathfrak{X}^{*}$. Then there exists a $\left[2(1+\sqrt{2})^{2}+\varepsilon\right]$-bounded $c_{0^{-}}$ biorthogonal system $\left\{x_{n}, x_{n}^{*}\right\} \subset \mathfrak{X} \times \mathfrak{X}^{*}$ with $\left[a_{n}\right]_{n} \subseteq\left[x_{n}\right]_{n}$ and $\left[b_{n}^{*}\right]_{n} \subseteq\left[x_{n}^{*}\right]_{n}$.

Proof. Without loss of generality, $\left[a_{n}\right]_{n \in \mathbb{N}}$ and $\left[b_{n}^{*}\right]_{n \in \mathbb{N}}$ are each infinitedimensional. Since $c_{0} \hookrightarrow \mathfrak{X}$, by Theorem 4.6, there is a $(2+\varepsilon)$-bounded biorthogonal system $\left\{w_{n}, w_{n}^{*}\right\}$ in $S(\mathfrak{X}) \times \mathfrak{X}^{*}$ with $\sum_{n} w_{n}$ wuC. Fix a sequence $\left\{\delta_{k}\right\}_{k=1}^{\infty}$ of positive numbers decreasing to zero with $\sum_{k} \delta_{k}<\infty$. Again we follow the notation in Definition 3.8. It suffices to find a system $\left\{x_{n}, x_{n}^{*}\right\}_{n=1}^{\infty}$ in $\mathfrak{X} \times \mathfrak{X}^{*}$ along with a blocking $\left\{J_{k}\right\}_{k=1}^{\infty}$ of $\mathbb{N}$ and an increasing sequence $\left\{i_{n}\right\}_{n \in \mathbb{N} o}$ from $\mathbb{N}$, satisfying

(a) $x_{m}^{*}\left(x_{n}\right)=\delta_{m n}$,

(b) $\left\|x_{n}\right\| \leq(1+\sqrt{2})+\varepsilon$,

(c) $\left\|x_{n}^{*}\right\| \leq(2+\varepsilon)(1+\sqrt{2})+\varepsilon$,

(d) for each $x^{*} \in S\left(\mathfrak{X}^{*}\right)$, if $n \in J_{k}$ then

$$
\left|x^{*}\left(x_{n}\right)\right| \leq(2+\sqrt{2}) \delta_{k}+(1+\sqrt{2}) \max _{j \in J_{k}^{o}}\left|x^{*}\left(w_{i_{j}}\right)\right|,
$$

(e) $\left[a_{n}\right]_{n=1}^{\infty} \subset\left[x_{n}\right]_{n=1}^{\infty}$,

(f) $\left[b_{n}^{*}\right]_{n=1}^{\infty} \subset\left[x_{n}^{*}\right]_{n=1}^{\infty}$. 
The construction will inductively produce blocks $\left\{x_{n}, x_{n}^{*}\right\}_{n \in J_{k}}$. Let $x_{0}$ and $x_{0}^{*}$ be the zero vectors. Fix $k \geq 1$. Assume that $\left\{J_{j}\right\}_{0 \leq j<k}$ along with $\left\{x_{n}, x_{n}^{*}\right\}_{n \in J_{k}^{p}}$ and $\left\{i_{n}\right\}_{n \in J_{k}^{p o}}$ have been constructed to satisfy conditions (a) through (d). Now to construct $J_{k}$ along with $\left\{x_{n}, x_{n}^{*}\right\}_{n \in J_{k}}$ and $\left\{i_{n}\right\}_{n \in J_{k}^{o}}$.

Let

$$
\mathcal{P}_{k}:=\left[x_{n}^{*}\right]_{n \in J_{k}^{p}}^{\top}, \quad \mathcal{Q}_{k}:=\left[x_{n}\right]_{n \in J_{k}^{p}}^{\perp}, \quad n_{k}=\max J_{k}^{p} .
$$

The idea is to find a biorthogonal system $\left\{z_{n}, z_{n}^{*}\right\}_{n \in J_{k}}$ in $\mathcal{P}_{k} \times \mathcal{Q}_{k}$ by first finding just one pair $\left\{z_{1+n_{k}}, z_{1+n_{k}}^{*}\right\}$ which helps guarantee condition (e) if $k$ is odd and condition (f) if $k$ is even; however, $\left\{z_{1+n_{k}}, z_{1+n_{k}}^{*}\right\}$ would not necessarily satisfy conditions (b) through (d) so $J_{k}^{o}$ and $\left\{z_{n}, z_{n}^{*}\right\}_{n \in J_{k}^{o}}$, and $\left\{i_{n}\right\}_{n \in J_{k}^{o}}$ are constructed and then the appropriate Haar matrix is applied to $\left\{z_{n}, z_{n}^{*}\right\}_{n \in J_{k}}$ to produce $\left\{x_{n}, x_{n}^{*}\right\}_{n \in J_{k}}$ so that $\left\{x_{n}, x_{n}^{*}\right\}_{n \in J_{k}^{p} \cup J_{k}}$ with $\left\{i_{n}\right\}_{n \in J_{k}^{p o} \cup J_{k}^{o}}$ satisfy conditions (a) through (d).

The pair $\left\{z_{1+n_{k}}, z_{1+n_{k}}^{*}\right\}$ is constructed by a standard Gram-Schmidt biorthogonal procedure. If $k$ is odd, start in $\mathfrak{X}$. Let

$$
h_{k}=\min \left\{h: a_{h} \notin\left[x_{n}\right]_{n \leq n_{k}}\right\} .
$$

Set

$$
z_{1+n_{k}}=a_{h_{k}}-\sum_{n \leq n_{k}} x_{n}^{*}\left(a_{h_{k}}\right) x_{n}
$$

and for any $y_{1+n_{k}}^{*}$ in $\mathfrak{X}^{*}$ such that $y_{1+n_{k}}^{*}\left(z_{1+n_{k}}\right) \neq 0$,

$$
z_{1+n_{k}}^{*}=\frac{y_{1+n_{k}}^{*}-\sum_{n \leq n_{k}} y_{1+n_{k}}^{*}\left(x_{n}\right) x_{n}^{*}}{y_{1+n_{k}}^{*}\left(z_{1+n_{k}}\right)} .
$$

If $k$ is even, start in $\mathfrak{X}^{*}$. Let

$$
h_{k}=\min \left\{h: b_{h}^{*} \notin\left[x_{n}^{*}\right]_{n \leq n_{k}}\right\} .
$$

Set

$$
z_{1+n_{k}}^{*}=b_{h_{k}}^{*}-\sum_{n \leq n_{k}} b_{h_{k}}^{*}\left(x_{n}\right) x_{n}^{*}
$$

and, for any $y_{1+n_{k}}$ in $\mathfrak{X}$ such that $z_{1+n_{k}}^{*}\left(y_{1+n_{k}}\right) \neq 0$,

$$
z_{1+n_{k}}=\frac{y_{1+n_{k}}-\sum_{n \leq n_{k}} x_{n}^{*}\left(y_{1+n_{k}}\right) x_{n}}{z_{1+n_{k}}^{*}\left(y_{1+n_{k}}\right)} .
$$

Clearly $z_{1+n_{k}}^{*}\left(z_{1+n_{k}}\right)=1, z_{1+n_{k}} \in \mathcal{P}_{k}$ and $z_{1+n_{k}}^{*} \in \mathcal{Q}_{k}$.

Find a natural number $m_{k}$ larger than one so that

$$
2^{-m_{k} / 2} \max \left(\left\|z_{1+n_{k}}\right\|,\left\|z_{1+n_{k}}^{*}\right\|\right)<\min \left(\varepsilon, \delta_{k}\right)
$$

and let

$$
J_{k}:=\left\{1+n_{k}, \ldots, 2^{m_{k}}+n_{k}\right\} \quad \text { and so } \quad J_{k}^{o}:=\left\{2+n_{k}, \ldots, 2^{m_{k}}+n_{k}\right\} .
$$


Let

$$
\widetilde{\mathcal{P}}_{k}:=\mathcal{P}_{k} \cap\left[z_{1+n_{k}}^{*}\right]^{\top}, \quad \widetilde{\mathcal{Q}}_{k}:=\mathcal{Q}_{k} \cap\left[z_{1+n_{k}}\right]^{\perp} .
$$

Now we find a biorthogonal system $\left\{z_{n}, z_{n}^{*}\right\}_{n \in J_{k}^{o}}$ along with $\left\{i_{n}\right\}_{n \in J_{k}^{o}}$ satisfying

$$
\left\{z_{n}, z_{n}^{*}\right\} \in S\left(\widetilde{\mathcal{P}}_{k}\right) \times(2+\varepsilon) B\left(\widetilde{\mathcal{Q}}_{k}\right)
$$

and

$$
\left\|w_{i_{n}}-z_{n}\right\|<\delta_{k}
$$

for each $n \in J_{k}^{o}$. Towards this, fix $j \in J_{k}^{o}$ and assume that a biorthogonal system $\left\{z_{n}, z_{n}^{*}\right\}_{2+n_{k} \leq n<j}$ along with $\left\{i_{n}\right\}_{2+n_{k} \leq n<j}$ have been constructed so that conditions (4.6) and (4.7) hold for $2+n_{k} \leq n<j$. Let

$$
\mathfrak{X}_{j}:=\widetilde{\mathcal{P}}_{k} \cap\left[z_{n}^{*}\right]_{2+n_{k} \leq n<j}^{\top}, \quad \mathcal{Y}_{j}:=\widetilde{\mathcal{Q}}_{k} \cap\left[z_{n}\right]_{2+n_{k} \leq n<j}^{\perp} .
$$

Apply Lemma 4.7 with $Y_{0}=\mathcal{Y}_{j}$ to get a finite-codimensional subspace $X_{0}$ of $\mathfrak{X}$ that is $(2+\varepsilon / 2)$-normed by $\mathcal{Y}_{j}$. Then by Fact 3.5 there exists a natural number $i_{j}>i_{j-1}$ along with $z_{j} \in S\left(\mathfrak{X}_{j} \cap X_{0}\right)$ such that

$$
d\left(w_{i_{j}}, \mathfrak{X}_{j} \cap X_{0}\right) \leq\left\|z_{j}-w_{i_{j}}\right\|<\delta_{k} .
$$

Since $X_{0}$ is $(2+\varepsilon / 2)$-normed by $\mathcal{Y}_{j}$ there is $\widetilde{z}_{j}^{*} \in S\left(\mathcal{Y}_{j}\right)$ such that

$$
\frac{1}{2+\varepsilon} \leq \widetilde{z}_{j}^{*}\left(z_{j}\right)
$$

Let

$$
z_{j}^{*}=\frac{1}{\widetilde{z}_{j}^{*}\left(z_{j}\right)} \widetilde{z}_{j}^{*}
$$

so that $z_{j}^{*}\left(z_{j}\right)=1$ and note that

$$
\left\|z_{j}^{*}\right\|=\frac{1}{\widetilde{z}_{j}^{*}\left(z_{j}\right)}\left\|\widetilde{z}_{j}^{*}\right\| \leq 2+\varepsilon .
$$

This completes the inductive construction of $\left\{z_{n}, z_{n}^{*}\right\}_{n \in J_{k}^{o}}$ and $\left\{i_{n}\right\}_{n \in J_{k}^{o}}$.

Now apply the Haar matrix to $\left\{z_{n}, z_{n}^{*}\right\}_{n \in J_{k}}$ to produce $\left\{x_{n}, x_{n}^{*}\right\}_{n \in J_{k}}$. With help from the observations in Remark 3.7, note that $\left\{x_{n}, x_{n}^{*}\right\}_{n \in J_{k}}$ is biorthogonal and is in $\mathcal{P}_{k} \times \mathcal{Q}_{k}$. Furthermore, for each $n$ in $J_{k}$,

$$
\begin{aligned}
& \left\|x_{n}\right\| \leq 2^{-m_{k} / 2}\left\|z_{1+n_{k}}\right\|+(1+\sqrt{2}) \max _{j \in J_{k}^{o}}\left\|z_{j}\right\| \leq \varepsilon+(1+\sqrt{2}) \\
& \left\|x_{n}^{*}\right\| \leq 2^{-m_{k} / 2}\left\|z_{1+n_{k}}^{*}\right\|+(1+\sqrt{2}) \max _{j \in J_{k}^{o}}\left\|z_{j}^{*}\right\| \leq \varepsilon+(2+\varepsilon)(1+\sqrt{2}) .
\end{aligned}
$$

If $x^{*} \in S\left(\mathfrak{X}^{*}\right)$ then

$$
\begin{aligned}
\left|x^{*}\left(x_{n}\right)\right| & \leq 2^{-m_{k} / 2}\left\|z_{1+n_{k}}\right\|+(1+\sqrt{2}) \max _{j \in J_{k}^{o}}\left|x^{*}\left(z_{j}\right)\right| \\
& \leq \delta_{k}+(1+\sqrt{2}) \max _{j \in J_{k}^{o}}\left(\left|x^{*}\left(z_{j}-w_{i_{j}}\right)\right|+\left|x^{*}\left(w_{i_{j}}\right)\right|\right)
\end{aligned}
$$




$$
\begin{aligned}
& \leq \delta_{k}+(1+\sqrt{2}) \max _{j \in J_{k}^{o}}\left(\delta_{k}+\left|x^{*}\left(w_{i_{j}}\right)\right|\right) \\
& =(2+\sqrt{2}) \delta_{k}+(1+\sqrt{2}) \max _{j \in J_{k}^{o}}\left|x^{*}\left(w_{i_{j}}\right)\right| .
\end{aligned}
$$

Thus $\left\{x_{n}, x_{n}^{*}\right\}_{n \in J_{k}^{p} \cup J_{k}}$ with $\left\{i_{n}\right\}_{n \in J_{k}^{p o} \cup J_{k}^{o}}$ satisfy conditions (a) through (d). If $k$ is odd, then

$$
\left[a_{h}\right]_{h \leq h_{k}} \subset\left[x_{n}, z_{1+n_{k}}\right]_{n \in J_{k}^{p}} \subset\left[x_{n}\right]_{n \in J_{k}^{p} \cup J_{k}},
$$

while if $k$ is even, then

$$
\left[b_{h}^{*}\right]_{h \leq h_{k}} \subset\left[x_{n}^{*}, z_{1+n_{k}}^{*}\right]_{n \in J_{k}^{p}} \subset\left[x_{n}^{*}\right]_{n \in J_{k}^{p} \cup J_{k}} .
$$

Clearly the constructed system $\left\{x_{n}, x_{n}^{*}\right\}_{n=1}^{\infty}$ with the blocking $\left\{J_{k}\right\}_{k=1}^{\infty}$ of $\mathbb{N}$ and the increasing sequence $\left\{i_{n}\right\}_{n \in \mathbb{N}^{\circ}}$ from $\mathbb{N}$ satisfy conditions (a) through (f).

Note that condition (d) tells us that if for each $k \in \mathbb{N}$ we pick any $n_{k} \in J_{k}$, then for $x^{*} \in S\left(\mathfrak{X}^{*}\right)$ we have

$$
\begin{aligned}
\sum_{k}\left|x^{*}\left(x_{n_{k}}\right)\right| & \leq(2+\sqrt{2}) \sum_{k} \delta_{k}+(1+\sqrt{2}) \sum_{k} \max _{j \in J_{k}^{o}}\left|x^{*}\left(w_{i_{j}}\right)\right| \\
& \leq(2+\sqrt{2}) \sum_{k} \delta_{k}+(1+\sqrt{2}) \sum_{j}\left|x^{*}\left(w_{i_{j}}\right)\right|<\infty .
\end{aligned}
$$

So $\sum_{k} x_{n_{k}}$ is wuC.

5. Piecing it all together. Inspired by Theorem 2.3 we might try to combine Theorems 3.3 and 4.5 with the Dilworth-Girardi-Johnson $\ell_{1}$ result (Theorem 2.4) to get the following theorem giving the existence of biorthogonal systems in any Banach space.

FAlse Conjecture 5.1. For any given infinite-dimensional Banach space $\mathfrak{X}$ there exists a bounded biorthogonal system $\left\{x_{n}, x_{n}^{*}\right\}$ that is of one of the following three types:

(1) a co-biorthogonal system,

(2) a wc $c_{0}^{*}$-stable biorthogonal system,

(3) a DP-biorthogonal system.

However, this does not follow directly from the previous results. The trouble lies in part (3). Theorem 2.3 guarantees us that if $\mathfrak{X}$ contains no isomorphic copies of $c_{0}$ or $\ell_{1}$, then there is a subspace (say $\mathcal{Y}$ ) of $\mathfrak{X}$ that fails DP. So from Theorem 3.3 we get a DP-biorthogonal system $\left\{y_{n}, y_{n}^{*}\right\}$ in $\mathcal{Y} \times \mathcal{Y}^{*}$. Since $\left\{y_{n}\right\}_{n}$ is weakly null in $\mathcal{Y}$ it is also weakly null in $\mathfrak{X}$. Unfortunately the fact that $\left\{y_{n}^{*}\right\}_{n}$ is weakly null in $\mathcal{Y}^{*}$ does not necessarily tell us that if we extend each $y_{n}^{*}$ to $x_{n}^{*} \in \mathfrak{X}^{*}$, then $\left\{x_{n}^{*}\right\}_{n}$ is weakly null in $\mathfrak{X}^{*}$. Another way to see that part (3) is not correct is to notice that DP does not necessarily pass to closed subspaces. Since it is a $C(K)$ space, $\ell_{\infty}$ has 
DP; however $\ell_{2}$ does not have DP. So if part (3) were correct it would say that $\mathcal{Y}$ failing DP implies $\mathfrak{X}$ fails DP, which is false. We recall the following related property.

Definition 5.2. A Banach space $\mathfrak{X}$ has the hereditary Dunford-Pettis property $\left(\mathrm{DP}_{\mathrm{h}}\right)$ if every closed subspace of $\mathfrak{X}$ has the Dunford-Pettis property.

For detailed discussions of $\mathrm{DP}_{\mathrm{h}}$ see $[2,3,4]$. In 1987 Cembranos gave the following useful characterization of $\mathrm{DP}_{\mathrm{h}}$.

Theorem 5.3 ([3]). A Banach space $\mathfrak{X}$ has $\mathrm{DP}_{\mathrm{h}}$ if and only if every normalized weakly null sequence in $\mathfrak{X}$ has a subsequence which is equivalent to the unit vector basis of $c_{0}$.

In 1989 Knaust and Odell [9] gave a quantitative improvement of this result by showing that the equivalence is uniform for all normalized weakly null sequences. Using the hereditary Dunford-Pettis property we can restate Theorem 2.3.

Restatement 5.4. Every infinite-dimensional Banach space, $\mathfrak{X}$, contains a subspace isomorphic to $c_{0}$, a subspace isomorphic to $\ell_{1}$ or $\mathfrak{X}$ fails $\mathrm{DP}_{\mathrm{h}}$.

In light of this restatement we see that a biorthogonal system characterization of $\mathrm{DP}_{\mathrm{h}}$ is in order. Theorem 5.3 will give it to us.

Definition 5.5. A biorthogonal system $\left\{x_{n}, x_{n}^{*}\right\}$ in $\mathfrak{X} \times \mathfrak{X}^{*}$ is called a $\mathrm{DP}_{\mathrm{h}}$-biorthogonal system if $\left\{x_{n}\right\}_{n}$ is semi-normalized, weakly null and for any subsequence $\left\{x_{n_{j}}\right\}_{j}$ the series $\sum_{j} x_{n_{j}}$ is not wuC.

TheOREm 5.6. A Banach space $\mathfrak{X}$ fails $\mathrm{DP}_{\mathrm{h}}$ if and only if for each $\varepsilon>0$ there is a $(2+\varepsilon)$-bounded $\mathrm{DP}_{\mathrm{h}}$-biorthogonal system $\left\{x_{n}, x_{n}^{*}\right\}$ in $S(\mathfrak{X}) \times \mathfrak{X}^{*}$.

Proof. $(\Rightarrow)$ Suppose $\mathfrak{X}$ fails $\mathrm{DP}_{\mathrm{h}}$ and $\varepsilon>0$. Then Theorem 5.3 gives us a normalized weakly null sequence $\left\{x_{n}\right\}_{n}$ with no subsequence equivalent to the unit vector basis of $c_{0}$. Without loss of generality $\left\{x_{n}\right\}_{n}$ is a basic sequence with basis constant at most $2+\varepsilon$. Now if for some subsequence $\left\{x_{n_{j}}\right\}_{j}$ we have $\sum_{j} x_{n_{j}}$ wuC then Theorem 4.2 tells us that $\left\{x_{n_{j}}\right\}_{j}$ is equivalent to the unit vector basis of $c_{0}$, which is a contradiction. Since $\left\{x_{n}\right\}_{n}$ is basic with basis constant at most $2+\varepsilon$, we may pick a sequence of biorthogonal functionals $\left\{x_{n}^{*}\right\}_{n} \subset(2+\varepsilon) B\left(\mathfrak{X}^{*}\right)$.

$(\Leftarrow)$ Suppose there exists such a biorthogonal system $\left\{x_{n}, x_{n}^{*}\right\}$. If $\mathfrak{X}$ has $\mathrm{DP}_{\mathrm{h}}$ then Theorem 5.3 gives us a subsequence $\left\{x_{n_{j}}\right\}_{j}$ of $\left\{x_{n}\right\}_{n}$ that is equivalent to the unit vector basis of $c_{0}$. But then we would have $\sum_{j} x_{n_{j}} \mathrm{wuC}$, which is a contradiction.

Finally, putting this together with Theorems 3.3 and 4.5 and the Dilworth-Girardi-Johnson $\ell_{1}$ result we get a correct theorem. 
THEOREM 5.7. For any given infinite-dimensional Banach space $\mathfrak{X}$ there exists a bounded biorthogonal system $\left\{x_{n}, x_{n}^{*}\right\}$ that is of one of the following three types:

(1) a co-biorthogonal system,

(2) a wc $c_{0}^{*}$-stable biorthogonal system,

(3) a $\mathrm{DP}_{\mathrm{h}}$-biorthogonal system.

Note that this theorem confirms the importance of $c_{0}$ in infinite-dimensional Banach spaces. The presence of a $c_{0}$-biorthogonal system $\left\{x_{n}, x_{n}^{*}\right\}$ in $\mathfrak{X}$ gives us a part of $\mathfrak{X}$ which is particularly $c_{0}$-rich in the sense that $\left[x_{n}\right]$ is isomorphic to $c_{0}$ by design and, of course, the same is true for any subsequence $\left\{x_{n_{j}}\right\}_{j=1}^{\infty}$. On the other hand, the existence of a $\mathrm{DP}_{\mathrm{h}}$-biorthogonal system $\left\{x_{n}, x_{n}^{*}\right\}$ in $\mathfrak{X}$ would signify a part of $\mathfrak{X}$ is completely lacking in $c_{0}$ subspaces. In particular, $\left[x_{n}\right]$ is not isomorphic to $c_{0}$ and the same is true for any subsequence $\left\{x_{n_{j}}\right\}_{j=1}^{\infty}$ since $\sum_{n} x_{n_{j}}$ is not wuC. In the third case if $\mathfrak{X}$ has a $w c_{0}^{*}$-stable biorthogonal system $\left\{x_{n}, x_{n}^{*}\right\}$, then $\left[x_{n}\right]$ is not isomorphic to $c_{0}$ since the proof in $[7]$ yields $\left[x_{n}\right] \approx \ell_{1}$.

It would be interesting to see what this interpretation of Theorem 5.7 yields in terms of other properties and structures that have been characterized using $c_{0}$. For instance, can we say anything about the existence of spreading models or nice (resp. not very nice) operators on the space?

\section{References}

[1] C. Bessaga and A. Pełczyński, On bases and unconditional convergence of series in Banach spaces, Studia Math. 17 (1958), 151-164.

[2] J. M. F. Castillo and M. Gonzáles, On the Dunford-Pettis property in Banach spaces, Acta Univ. Carolin. Math. Phys. 35 (1994), no. 2, 5-12.

[3] P. Cembranos, The hereditary Dunford-Pettis property on $C(K, E)$, Illinois J. Math. 31 (1987), 365-373.

[4] J. Diestel, A survey of results related to the Dunford-Pettis property, in: Proc. Conf. of Integration, Topology, and Geometry in Linear Spaces (Chapel Hill, NC, 1979), Contemp. Math. 2, Amer. Math. Soc., 1980, Providence, RI, 15-60.

[5] - Sequences and Series in Banach Spaces, Grad. Texts Math. 92, Springer, New York, 1984.

[6] J. Diestel and J. J. Uhl, Jr., Vector Measures, with a foreword by B. J. Pettis, Math. Surveys 15, Amer. Math. Soc., Providence, RI, 1977.

[7] S. J. Dilworth, M. Girardi, and W. B. Johnson, Geometry of Banach spaces and biorthogonal systems, Studia Math. 140 (2000), 243-271.

[8] P. Enflo, A counterexample to the approximation problem in Banach spaces, Acta Math. 130 (1973), 309-317.

[9] H. Knaust and E. Odell, On co sequences in Banach Spaces, Israel J. Math. 67 (1989), 153-169.

[10] J. Lindenstrauss and L. Tzafriri, Classical Banach Spaces. I: Sequence Spaces, Ergeb. Math. Grenzgeb. 92, Springer, Berlin, 1977. 
[11] A. I. Markushevich, On a basis in the wide sense for linear spaces, Dokl. Akad. Nauk SSSR 41 (1943), 241-244.

[12] E. Odell, Applications of Ramsey theorems to Banach space theory, in: Notes in Banach Spaces, Univ. of Texas Press, Austin, TX, 1980, 379-404.

Department of Mathematics

Lynchburg College

Lynchburg, VA 24501, U.S.A.

E-mail: coco@lynchburg.edu

Received December 16, 2003

Revised version April 26, 2004 